\title{
Localization of vasa, a component of Drosophila polar granules, in maternal- effect mutants that alter embryonic anteroposterior polarity
}

\author{
BRUCE HAY, LILY YEH JAN and YUH NUNG JAN \\ Howard Hughes Medical Institute, Departments of Physiology and Biochemistry, University of California, San Francisco, CA 94143, USA
}

\section{Summary}

Cytoplasm at the posterior pole of the early Drosophila embryo, known as polar plasm, serves as a source of information necessary for germ cell determination and for specification of the abdominal region. Likely candidates for cytoplasmic elements important in one or both of these processes are polar granules, organelles concentrated in the cortical cytoplasm of the posterior pole. Females homozygous for any one of the maternal-effect mutations, tudor, oskar, staufen, vasa, or valois give rise to embryos that lack localized polar granules, fail to form the germ cell lineage and have abdominal segment deletions. Using antibodies against a polar granule component, the vasa protein, we find that vasa synthesis or localization is affected by these mutations. In vasa mutants, synthesis of vasa protein is absent or severely restricted. In oskar and staufen mutant females, vasa synthesis appears normal, but the vasa protein is not localized. In tudor and valois mutant females, vasa is localized to the posterior pole of oocytes, but this localization is lost following egg activation. In addition to the posterior localized vasa, there is a low level of vasa distributed throughout the embryo. A function for this distributed vasa is postulated based on the observation that embryos from Bicaudal-D mothers, in which abdominal determinants are incorrectly localized to the anterior pole, do not show any ectopic vasa localization, though abdomen development at the anterior end depends on the amount of vasa protein in the embryo.

Key words: vasa, polar granules, maternal-effect mutants, cytoplasmic determinants.

\section{Introduction}

Asymmetric segregation of cytoplasmic components is observed during early stages of embryogenesis of many vertebrate and invertebrate embryos (Davidson, 1986). One well-known example is the polar plasm of Drosophila, which contains large numbers of organelles called polar granules (Mahowald, 1962, 1968). Polar granules are electron-dense, RNA-rich cytoplasmic organelles, and are concentrated to the posterior pole of late stage oocytes and early embryos (Mahowald, 1968, $1971 b)$. Several lines of evidence indicate that these structures are required for the localization and/or function of posteriorly localized cytoplasmic determinants required for the formation of the germ cell lineage and for proper abdomen specification. First, mutations of vasa, which codes for a component of polar granules, abolish the formation of germ cells and alter abdominal segment specification (Schüpbach and Wieschaus, 1986; Hay et al. 1988a,b; Lasko and Ashburner, 1988). Secondly, six other maternal-effect mutations (tudor, Boswell and Mahowald, 1985; Schüpbach and Wieschaus, 1986; staufen, valois, Schüpbach and Wieschaus, 1986; oskar, Lehmann and Nüsslein-Volhard, 1986; and cappuccino and spire, Manseau and Schüpbach, 1989), which disrupt formation of the germ cell lineage and abdomen specification, also produce embryos without localized polar granules. Finally, the temporal distribution of RNA-rich polar granules in wild-type oocytes and embryos coincides with the ability of polar plasm to induce germ cell determination (Mahowald, 1971b; Illmensee et al. 1976; Niki, 1986).

Synthesis of polar granule components and their localization to the posterior embryonic pole occurs during oogenesis. Assembly of the granule probably occurs in the nurse cells, germline feeder cells, because these cells contain large cytoplasmic masses (nuage) which resemble polar granules, and which contain an identified component of polar granules, the product of the vasa locus (Hay et al. 1988a). During the second half of oogenesis (stages 10-14 of King, 1970), the nurse cells inject essentially all of their cytoplasmic contents into the growing oocyte through intercellular bridges located at the anterior pole of the oocyte. Cytoplasmic mixing rapidly distributes this material throughout the ooplasm (Gutzeit and Koppa, 1982). This is the period during which localization of polar granules to the posterior pole occurs (Mahowald, 1971a). This posterior localization, in spite of entry at the anterior pole and rapid mixing, probably involves specific transport mechanisms which target polar granules to the posterior pole. Maintenance of this posterior localization may in 
turn require specific interactions between receptors at the posterior pole and certain polar granule components.

To understand the requirements for polar granule localization and to identify stages involved in this process, one may follow the fate of known granule components in mutants that lack localized polar granules. Here we report experiments in which we have followed the fate of the vasa protein, a component of polar granules, during oogenesis and embryogenesis in a number of mutants. Implications of vasa function and of mechanisms of granule localization will be discussed.

\section{Materials and methods}

\section{Stocks}

Drosophila melanogaster were raised on standard cornmeal-yeast-agar medium at $25^{\circ} \mathrm{C}$. Normal flies were of Oregon-R (OR) wildtype strain. Balanced stocks of vasa (vasa ${ }^{\mathrm{DD} 23}$ and vasa ${ }^{\mathrm{DL}}$ ), tudor (tudor ${ }^{\mathrm{WCO}}$ ), a deficiency that uncovers the tudor locus (Df(2L)PL3), staufen, staufen ${ }^{\text {HLS4 }}$, a deficiency that uncovers the staufen locus (Df(2L) PC4), valois, (valois $\left.{ }^{\mathrm{RB}}\right)$ and a deficiency that uncovers the valois locus ( $\operatorname{Df}(2 \mathrm{~L}) \mathrm{TW} 2)$, were provided by Trudi Schüpbach, Princeton University, Princeton, NJ. Other stocks of tudor

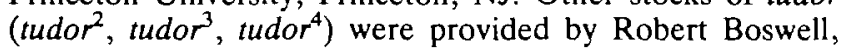
University of Colorado, Boulder, CO. Stocks of the two deficiencies $\operatorname{Df}(2 \mathrm{~L}) \mathrm{A} 267$ and $\mathrm{Df}(2 \mathrm{~L}) \mathrm{TE} 116-\mathrm{GW} 18$, which in heterozygous combination delete part of the vasa locus, staufen $\left(\right.$ staufen $\left.^{\mathrm{D} 3}\right)$, oskar (oskar ${ }^{108}$, oskar ${ }^{336}$, oskar $\left.{ }^{301}\right)$, a deficiency that uncovers the oskar locus, stocks of valois $\left(\right.$ valois $\left.^{\mathrm{PE}}\right)$ and stocks of Bicaudal-D (Bicaudal- $)^{7134}, B i$ caudal- $D^{\text {IIIE }}$ ) were provided by Ruth Lehmann, Whitehead Institute, Cambridge, MA.

\section{Fly crosses}

For most experiments, homozygous females were collected from balanced stocks and mated with $\mathrm{OR}$ males. For the creation of a deletion of a part of the vasa locus, virgin $\mathrm{Df}(2 \mathrm{~L}) \mathrm{A} 267 / \mathrm{CyO}$ females, which are deleted for a part of the vasa locus, were crossed to $\mathrm{Df}(2 \mathrm{~L}) \mathrm{TE} 116-\mathrm{GW} 18 / \mathrm{CyO}$ males, which are also deleted for a part of the vasa locus, and non$\mathrm{CyO}$ progeny selected. The only transcription unit affected by both deficiencies in these flies is vasa (Lasko and Ashburner, 1988).

\section{Production of polyclonal anti-vasa rabbit serum}

A trpE-vasa fusion protein was used as the immunogen. Site directed mutagenesis was carried out to create an NdeI restriction site at the initial methionine of a full-length vasa cDNA cloned into bluescript SK- (Stratagene) as described (Kunkel et al. 1987). A pair of oligonucleotides was synthesized, phosphorylated and annealed using standard techniques (Maniatis et al. 1982) such that BamHI and NdeI sites were created as opposite ends of the oligonucleotide. A gelpurified NdeI-XbaI fragment containing the entire vasa coding region was mixed with these oligonucleotides and ligated into the vector PATH1, cut with BamHI and XbaI. This vector contains the trpE coding sequence (Dieckmann and Tzagaloff, 1985). Bacteria containing either a trpE-vasa construct or $\operatorname{trp} E$ alone were grown and induced according to Driever $e$ t al. (1988). Fusion protein was isolated according to Rio et al. (1986), dissolved in sample buffer (Laemmli, 1970) and subjected to preparative SDS-PAGE. Protein bands were visualized by incubating the gel with $0.25 \mathrm{M} \mathrm{KCl}$ in $\mathrm{H}_{2} \mathrm{O}$. The gel slice containing the trpE-vasa fusion protein was cut out and chopped into small bits. These were then suspended in complete Freund's adjuvant (incomplete for booster injections). Rabbits (Berkeley Antibody Company, Berkeley, CA) were injected at multiple subcutaneous and intramuscular sites, boosted after 4 weeks, and bled at biweekly intervals thereafter. Two rabbits produced high titers against the vasa protein. To purify antibodies specific for the vasa portion of the fusion protein, the following procedure was carried out. Bacterial extract from cells expressing either the trpE-vasa protein or $\operatorname{trp} E$ alone were dialyzed into coupling buffer $(0.1 \mathrm{M}$ Hepes (pH7.5), 10\% glycerin, $5 \mathrm{~mm}$ EDTA). Precipitated material was sedimented at $10000 \mathrm{~g}, 10 \mathrm{~min}$. The supernatants were used to prepare affinity columns (Affigel 10/15, 1:1 ratio; Biorad) according to the manufacturer's protocol. Following coupling, these columns were washed exhaustively with $0.5 \mathrm{M} \mathrm{NaCl}, 10 \mathrm{M}$ Tris- $\mathrm{HCl}, \mathrm{pH} 7.5$, and then with elution buffer containing $0.2 \mathrm{M}$ glycine, $0.15 \mathrm{M} \mathrm{HCl}, 0.5 \%$ Tween-20, $\mathrm{pH}$ 3.5. The column was then equilibrated with antibodybinding buffer ( $50 \mathrm{~mm}$ Tris-HCl, pH 7.5, $5 \mathrm{~mm}$ EDTA). Rabbit serum was dialyzed into antibody-binding buffer and then incubated overnight at $4^{\circ} \mathrm{C}$ batchwise with column material containing bacterial extract induced to express trpE alone. The unbound material was incubated batchwise for four hours at room temperature with affigel containing extract from the trpE-vasa expressing bacteria. This affigel was then poured into a column and washed with 50 column volumes of antibody-binding buffer. Bound protein was eluted with 3 column volumes of elution buffer. Elution volumes were immediately neutralized with $1 \mathrm{M}$ Tris base and brought to a final concentration of $1 \% \mathrm{BSA}, 0.02 \%$ sodium azide.

\section{Immunocytochemistry}

Antibody staining of different stages of oogenesis and embryogenesis with monoclonal antibody Mab46F11, which recognizes the product of the vasa locus, was carried out as described in Hay et al. (1988a). For immunocytochemistry using the polyclonal antisera, tissue was incubated for $1 \mathrm{~h}$ at room temperature with the affinity-purified anti-vasa antibodies at a dilution of $1: 1000$ in antibody buffer $(0.1 \mathrm{~m}$ sodium phosphate, $\mathrm{pH} 7.5,0.3 \%$ Triton $\mathrm{X}-100,0.3 \%$ sodium deoxycholate, $2 \%$ bovine serum albumin (Miles, fraction V)) Three, $15 \mathrm{~min}$ washes in antibody-binding buffer were followed by incubation of the tissue for $1 \mathrm{~h}$ at room temperature with horseradish peroxidase-conjugated goat anti-rabbit IgG (Biorad, EIA grade) at a dilution of 1: 1000. Following three $15 \mathrm{~min}$ washes as above, the diaminobenzidine reaction product was developed as described in Hay et al. (1988a). DNA was visualized by incubating embryos with $0.5 \mu \mathrm{g} \mathrm{ml}^{-1} 4^{\prime}, 6$ diamidine-2-phenylindole dihydrochloride (DAPI, Boehringer Mannheim Biochemicals) in $0.1 \mathrm{M}$ Tris $-\mathrm{HCl}$ for $10 \mathrm{~min}$ at room temperature and then washing for $10 \mathrm{~min}$ in Tris buffer alone. The embryos were then mounted in $80 \%$ glycerol, 0.1 M Tris-HCl, pH 9.0, $5 \%$ (wt/vol) $n$-propyl gallate.

\section{Western blotting}

For Western blotting, $1 \mathrm{~h}$ collections of embryos were dechorionated in bleach: water $(1: 1)$, rinsed extensively with water and staged under a dissecting microscope. Cleavage stage embryos were then processed as described in Hay et al. (1988a) for Western blotting using the monoclonal antibody Mab46F11.

\section{Oocyte activation}

Oocyte activation was carried out according to Mahowald 
et al. (1983). Ovaries from well-fed virgin females were hand dissected and activated by placing them in $50 \%$ Robb's medium for up to several hours. Removal of unactivated occytes and dechorionation was carried out by removing the Robb's medium and adding a $30 \%$ solution of commercial bleach for several minutes. The surviving activated oocytes were then fixed as above for embryos and devitellinized by hand. Antibody staining was as above.

\section{Results}

Embryos derived from females homozygous for strong alleles of the five posterior group genes, vasa, staufen, oskar, valois and tudor, all have abdominal deletions, fail to form germ cells and lack polar granules at the posterior embryo pole. To examine the effects of these mutations on granule localization, we used monoclonal and polyclonal antibodies against the vasa product, a component of polar granules, and carried out Western (immunoblot) analysis and immunocytochemical staining of oocytes and embryos derived from homozygous mutant mothers. Null, as well as weaker alleles of vasa, were first analyzed to verify the specificity of the antibodies, before the studies of the other mutations. In order to assess the severity of these mutations, we compared the phenotypes generated by homozygous mutant females with those arising from females carrying the same mutation on one chromosome and a deletion of the locus on the other chromosome.

vasa distribution in embryos and oocytes of the wildtype and of vasa mutants

In wild-type embryos, vasa immunoreactivity, as seen with monoclonal antibody Mab46F11, is concentrated in the form of polar granules at the posterior pole (Hay et al. $1988 a, b)$. Lower levels of immunoreactivity are also found throughout the embryo during cleavage stages (Hay et al. 1988b). Because the vasa protein appears to have many different cellular associations: as a part of polar granules during oogenesis and early embryogenesis, as a part of the nuage or dense bodies in the adult germline, and distributed throughout the somatic region of the preblastoderm embryo, we were concerned that in some situations the epitope of vasa recognized by monoclonal antibody Mab46F11 might be masked, and thus we would be missing some populations of vasa proteins in our immunocytochemical analysis. To avoid this potential difficulty in the interpretation of our immunocytochemical data, we immunized rabbits with a full-length vasa protein made as a fusion with the bacterial protein trpE (see Materials and methods) in order to generate polyclonal anti-vasa antibodies. Vasa immunoreactivity, as seen with the polyclonal antiserum, shows a similar pattern during early embryogenesis (Fig. 1A). The immunoreactivity seen with monoclonal or polyclonal antibodies, localized to the posterior pole and present at lower levels throughout the embryo, is absent in embryos from homozygous vasa ${ }^{\mathrm{PD}}$ females (a hypomorphic allele) (see Hay et al. 1988b, Fig. 4) (Fig. 1B). Thus, both antibodies appear specific for the vasa product during early embryogenesis. The specificity of these antibodies for the vasa product is further demonstrated by Western analysis of proteins extracted from vasa cleavage stage embryos (Hay et al. 1988a, Fig. 7) (Fig. 2).

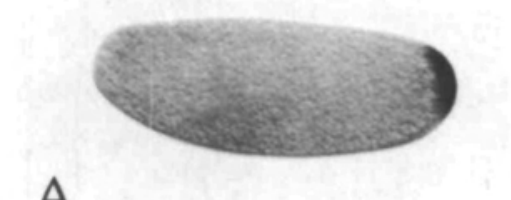

A
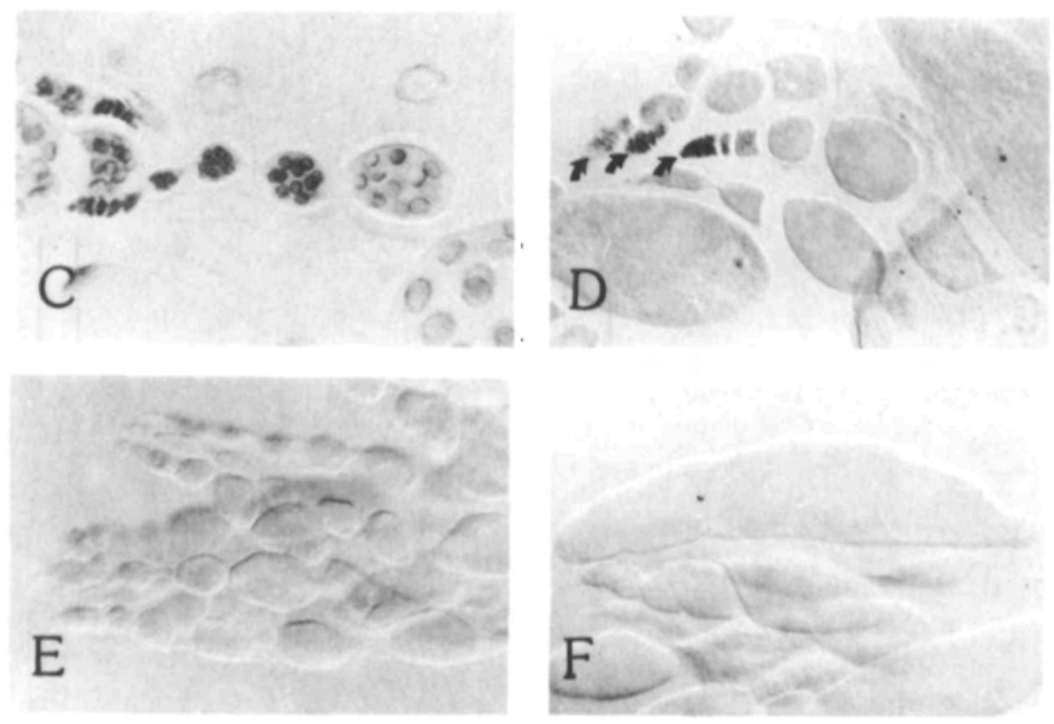



B

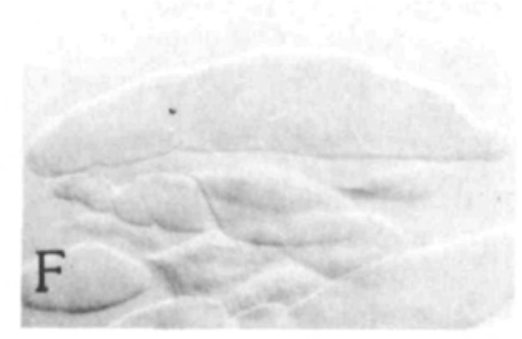

Fig. 1. Immunoreactivity in wild-type and vasa mutant ovaries as visualized using polyclonal rabbit anti-vasa antibodies. Younger stages of oogenesis, and the anterior of any given egg chamber are located to the left. (A) Cleavage stage wild-type embryo. Label is concentrated at the posterior embryo pole. A general background level of staining is present throughout the somatic regions of the embryo. (B) vasa ${ }^{\mathrm{PD}}$ cleavage stage embryo. No staining is present, at the posterior pole or throughout the rest of the embryo. (C) Wildtype ovary. (D) Homozygous vasa ${ }^{\mathrm{PD}}$ ovary. Label is primarily present in the germarium and early vitellarium stages (indicated by the arrows). (E) A vasa null ovary, which does not show any late stage oocytes. Label is undetectable. (F) A vasa null ovary, which does show a later stage oocyte (approximately stage 10). Label is not present. 


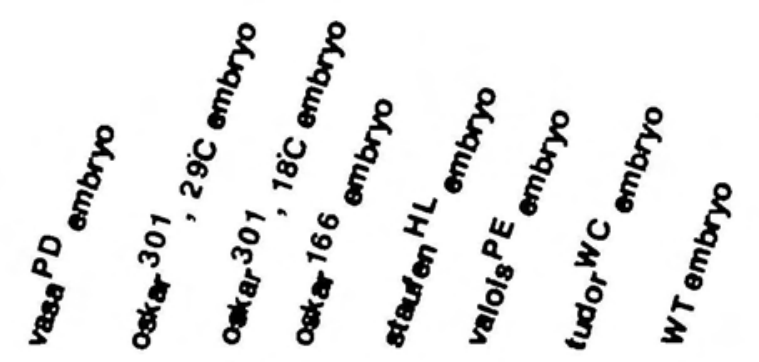

Fig. 2. Western blot of cleavage stage embryos from wildtype and homozygous mutant mothers for the mutations vasa, tudor, oskar, staufen, and valois. A $72 \times 10^{3} M_{\mathrm{r}}$ band is absent only in the vasa embryo lane. There is a lower molecular weight band present in all lanes whose identity is unknown. This band provides a convenient marker for relative gel loading (see also Hay et al. 1988a). Protein from cleavage stage embryos was prepared as in Hay et al. $(1988 a)$ and $10 \mu \mathrm{g}$ loaded per lane.

In ovaries of homozygous vasa $a^{\mathrm{PD}}$ females, vasa immunoreactivity is restricted to early stages, primarily the germarium, in which the geometry of the oocytenurse cell complex is set up. Occasionally, low levels of vasa immunoreactivity are also found in early stages of the vitellarium, in which the bulk of oocyte and nurse cell growth occurs (Fig. 1D; compare with wild type in Fig. 1C). In a female null for vasa function (see experimental methods for details), oogenesis is usually not completed. In ovaries of many such females, egg chambers do not develop beyond the early stages of the vitellarium (Fig. 1E), whereas in other ovaries egg chambers at later stages of oogenesis are present (up through stage 14) (Fig. 1F). None of these show any vasa immunoreactivity with either the monoclonal or polyclonal antibody. These observations show that our anti-vasa antibodies recognize only the vasa protein during oogenesis and early embryogenesis.

oskar and staufen mutations eliminate or reduce vasa localization during oogenesis

The oskar and staufen mutations affect vasa localization during oogenesis. Ovaries from strong (strength being defined relative to the amount of abdominal segment deletion seen) alleles of oskar and staufen, oskar ${ }^{36}$ and staufen ${ }^{\mathrm{D} 3}$ or these mutations in trans to deficiencies uncovering the respective loci, show a normal pattern of vasa expression in the nurse cells (Fig. 3A,B). Immunoreactivity appears in the oocyte around stage 10 , but never becomes localized to the posterior pole of stage 14 oocytes (Fig. 3C,D). Localization is also not seen in early cleavage stage embryos (Fig. 3E,F).

Western analysis reveals that normal levels of vasa product are present in early embryos of oskar ${ }^{166}$ (and oskar ${ }^{336}$, data not shown) or staufen ${ }^{\mathrm{D} 3}$ mothers (Fig. 2). This may derive from vasa synthesized by nurse cells and transported into the oocyte, or it may come from translation of vasa from the large amount of maternally derived vasa RNA present in the early embryo (Hay et al. 1988b). Although it remains possible that these mutations affected the stability of just the population of vasa that ends up at the posterior pole, from the fact that the absolute levels of vasa are not affected in these mutations, and in the absence of any information suggesting the existence of populations of vasa that differ in their stability, we favour the hypothesis that these mutations directly affect vasa localization. This phenotype is likely due to a reduction or loss of function, because a similar pattern of expression is seen immunocytochemically in oskar $r^{336}$ and staufen ${ }^{\mathrm{D} 3} \mathrm{fe}-$ males which carry the mutation on one chromosome and a deficiency for the region on the other chromosome.

A correlation between vasa localization and mutant phenotype is evident in a temperature-sensitive oskar mutant, os $k^{301}$. At $18^{\circ} \mathrm{C}$, the permissive temperature, many embryos show normal abdominal segmentation and hatch, though pole cells do not form. At $29^{\circ} \mathrm{C}$, they have strong abdominal deletions, in addition to the absence of pole cells (Lehmann and Nüsslein-Volhard, 1986). The degree of vasa localization in these embryos is correlated with the severity of the abdominal defect. At $18^{\circ} \mathrm{C}$, some vasa is found localized to the posterior pole of stage 10 oocytes, following oviposition weak localized staining is sometimes seen in cleavage stage embryos (Fig. 3G,H). Occasionally structures that resemble polar buds are formed, but these do not develop into pole cells and by blastoderm formation localized vasa immunoreactivity is undetectable. At the nonpermissive temperature, oskar ${ }^{301}$ resembles oskar ${ }^{336}$ with respect to its vasa localization phenotype (Fig. 3I,J).

valois and tudor mutations affect vasa localization during embryogenesis

The valois and tudor mutations tested affect vasa localization during early embryogenesis. Oocytes of homozygous valois ${ }^{\mathrm{RB} 71}$ or valois $\mathrm{PE}$, or valois $\mathrm{PE} /$ 


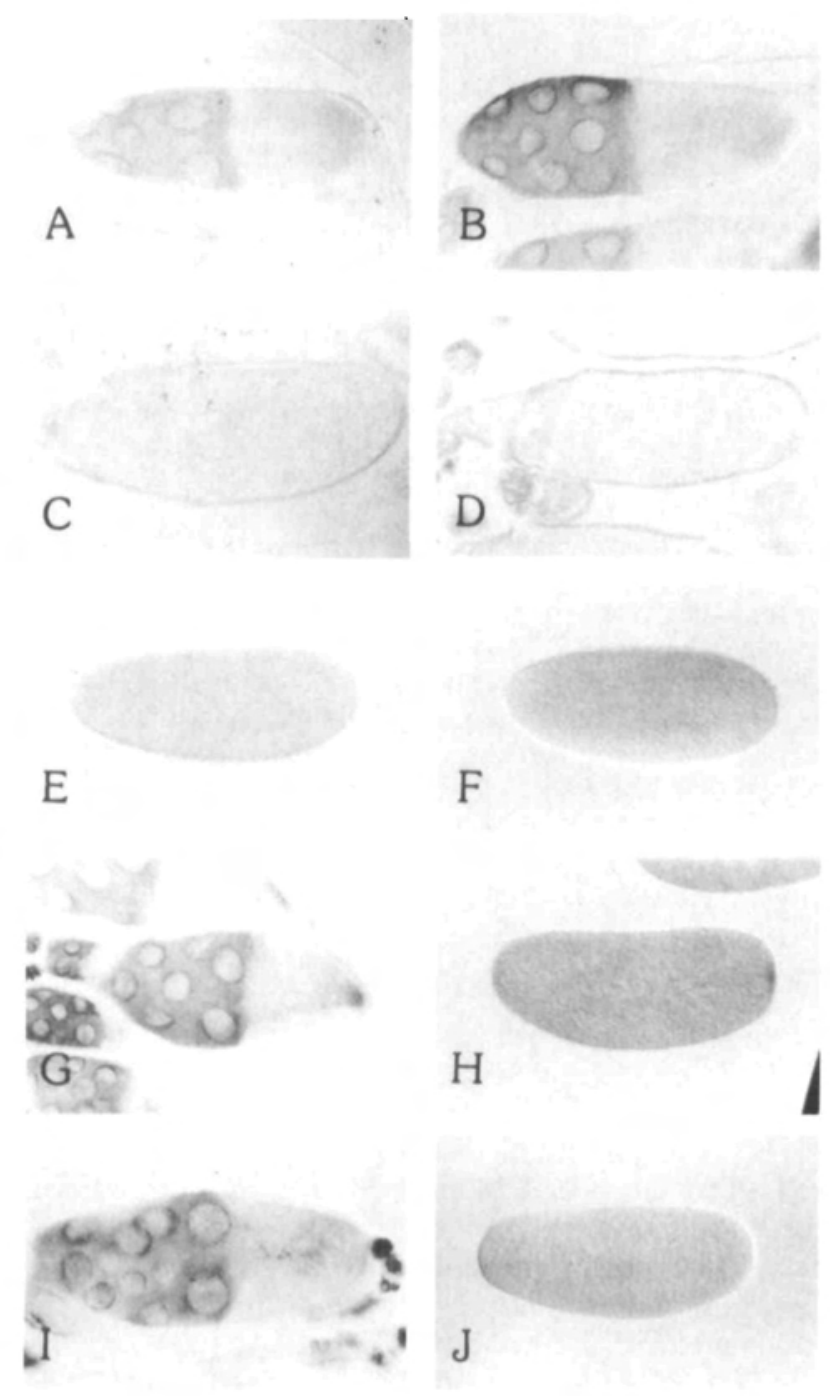

Fig. 3. vasa localization in stages of oogenesis and early embryos from homozygous oskar and staufen mothers. All antibody stainings were carried out with polyclonal antivasa antibodies. (A) Stage 10 oskar ${ }^{336}$ egg chamber. Label is present in nurse cells and in the oocyte but is not localized to the posterior oocyte pole. (B) Stage 10 staufen $^{\mathrm{D} 3}$ egg chamber. Label is present in nurse cells and oocyte but not localized to the posterior oocyte pole. (C) Cryostat section of an oskar ${ }^{336}$ stage 14 oocyte. Posterior label is not present. (D) Cryostat section of a staufen $^{\mathrm{D} 3}$ stage 14 oocyte. Posterior label is not present. (E) $o_{k a r}{ }^{336}$ early cleavage stage embryo. Localized label is not present. (F) staufen ${ }^{\mathrm{B}}$ early cleavage stage embryo. Localized label is not present. (G) Stage 10 oocyte from $o s k a r^{301}$ female raised at $18^{\circ} \mathrm{C}$. Some localized label is present at the posterior oocyte pole. $(\mathrm{H})$ Early cleavage stage embryo from oskar ${ }^{301}$ female raised at $18^{\circ} \mathrm{C}$. A small amount of label is localized to the posterior embryo pole. (I) Stage 10 oocyte from oskar ${ }^{301}$ female raised at $29^{\circ} \mathrm{C}$. Label is not detectably localized to the posterior oocyte pole. (J) Early cleavage stage embryo from oskar ${ }^{301}$ female raised at $29^{\circ} \mathrm{C}$. Label is not detectably localized to the embryo posterior pole.
Df(2L)TW2 females, have vasa localized to their posterior pole at stage 10 through stage 14 , as in the wildtype (Fig. 4A,B). However, following oviposition localized vasa immunoreactivity rapidly disappears, such that by the time of cellular blastoderm formation no localized immunoreactivity is present (Fig. 4C,D). Wild-type amounts of vasa immunoreactivity are present, however, by Western blotting (Fig. 2). When mutant embryos are also stained with DAPI to visualize their DNA, embryos with localized vasa show no more than several cleavage nuclei, whereas embryos that have proceeded through further cleavage divisions show no signs of localized vasa. This indicates that vasa localization is lost very rapidly following fertilization, long before pole cell formation would have occurred.

Embryos from homozygous tudor ${ }^{\mathrm{wC} 8}$ mothers have a very variable segmentation defect, with some embryos showing normal segmentation and others having abdominal deletions of varying size (Schüpbach and Wieschaus, 1986). Females carrying tudor ${ }^{\text {WCs }}$ on one chromosome and a deficiency for the region on the other consistently give rise to embryos with a stronger phenotype, indicating that tudor ${ }^{\mathrm{WC}}$ (as with other published tudor alleles) is probably a hypomorphic allele (Schüpbach and Wieschaus, 1986). In both the homozygous mutant and hemizygous situation, normal vasa localization and accumulation at the posterior pole is observed during the second half of oogenesis (Fig. 4E,F). Following oviposition, early cleavage stage embryos still have localized vasa immunoreactivity (Fig. 4G), whereas in later cleavage stage embryos, it becomes progressively weaker (Fig. $4 \mathrm{H})$. In some embryos, pole cell buds are observed to form (Fig. 4I), but these buds disappear by blastoderm formation (Fig. 4J,K). Localized vasa immunoreactivity is always absent by blastoderm formation (Fig. $4 \mathrm{~K}$ ), as is the general background seen during cleavage stages in wildtype and mutant embryos.

tudor ${ }^{\mathrm{W} C}$ embryos have some small electron-dense structures associated with mitochondria. These structures may be some form of polar granule. In a phenotypically weaker allele, such as $t u d o r^{2}$, more of these structures can be found and a more normal abdomen develops in the embryo (Boswell and Mahowald, 1985). Correspondingly, in these embryos localized vasa immunoreactivity is more intense and frequent, though this immunoreactivity still disappears prior to blastoderm formation.

\section{vasa localization in Bicaudal-D embryos}

In some of the embryos from mothers carrying the dominant bicaudal mutation Bicaudal-D, anterior structures are reduced or absent, and in extreme cases a posterior abdomen is duplicated in mirror-image symmetry to the normal abdomen, at the anterior end (Mohler and Wieschaus, 1986). At the transformed anterior end polar granules are not apparent and pole cells do not form, though in wild-type embryos a source of abdomen-inducing activity is colocalized at the posterior pole along with the germline determinants (Frohnhöfer et al. 1986; Lehmann and Nüsslein-Vol- 

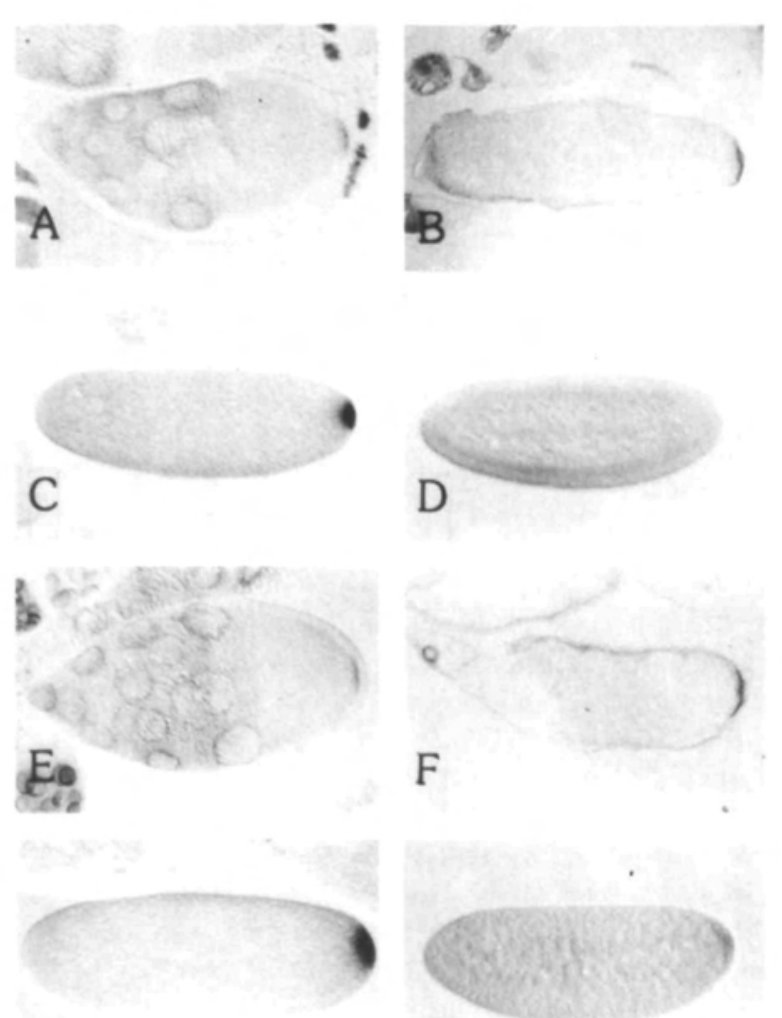

G
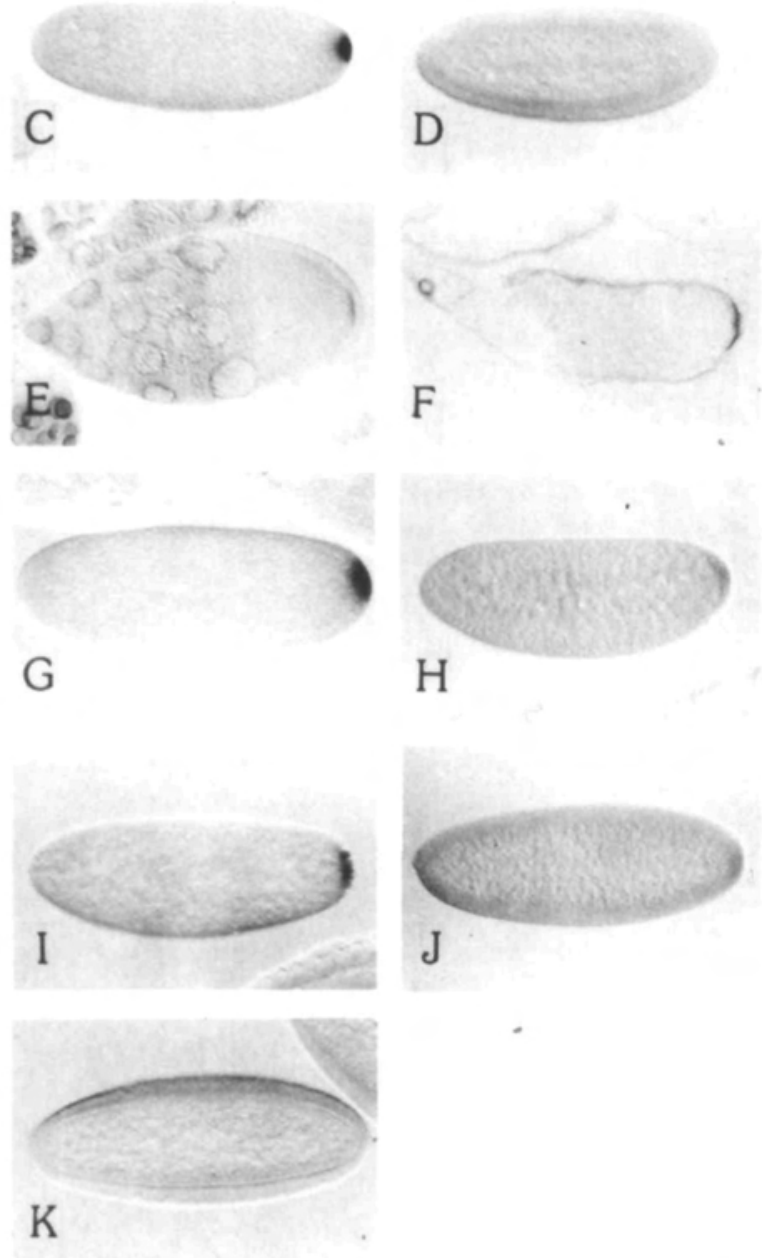

hard, 1986). The maternally encoded product of the nanos locus is likely to be the active component in the abdomen-inducing activity (Sander and Lehmann, 1988; Hülskamp et al. 1989; Irish et al. 1989; Struhl, 1989). Genetic and cytoplasmic transplantation experiments suggest that in Bicaudal-D, but not in wild-type embryos, nanos activity is localized at the anterior pole of the egg, as well as at the posterior pole (Lehmann and Nüsslein-Volhard, 1986; Wharton and Struhl, 1989). Interestingly, in Bicaudal-D females that are also heterozygous for vasa, the bicaudal phenotype is suppressed (Mohler and Wieschaus, 1986). The vasadependent suppression of the bicaudal phenotype indicates that vasa is required for the ectopic function of nanos activity. vasa-dependent suppression may occur during oogenesis or in the egg following fertilization. One possibility is that vasa is required for ectopic localization of the nanos product, for instance by
Fig. 4. vasa localization in stages of oogenesis and early embryogenesis from valois ${ }^{\mathrm{PE}} / \mathrm{Df}(2 \mathrm{~L}) \mathrm{TW} 2$ and

tudor $^{\text {wC }} / \mathrm{Df}(2 \mathrm{R}) \mathrm{PL} 3$ mothers. All antibody stainings were carried out with polyclonal anti-vasa antibodies. Similar observations were made with monoclonal antibody Mab46F11. (A) Stage 10 valois $^{\text {PE}} / \mathrm{Df}(2 \mathrm{~L}) \mathrm{TW} 2$ oocyte. Some label is localized to the oocyte posterior pole. (B) Cryostat section of a stage 14 valois $^{\mathrm{PE}} / \mathrm{Df}(2 \mathrm{~L}) \mathrm{TW} 2$ oocyte. A dense cortical band of label is present at the oocyte posterior pole. (C) Early cleavage stage valois $^{\mathrm{PE}} / \mathrm{Df}(2 \mathrm{~L}) \mathrm{TW} 2$ embryo, which shows some label at the posterior embryo pole. (D) Later cleavage stage valois $^{\mathrm{PE}} / \mathrm{Df}(2 \mathrm{~L}) \mathrm{TW} 2$ embryo with no localized posterior label. (E) Stage 10 tudor $^{\text {WC8 }} / \mathrm{Df}(2 \mathrm{R}) \mathrm{PL} 3$ oocyte. Localized label is present at the posterior oocyte pole. (F) Stage 14 tudor $^{\mathrm{wC} 8} / \mathrm{Df}(2 \mathrm{~L}) \mathrm{PL} 3$ oocyte. A dense band of label is present at the posterior oocyte pole. (G) Early cleavage stage tudor ${ }^{\text {WC8 }} /$ Df(2R)PL3 embryo. Large amounts of label are localized to the embryo posterior pole. $(\mathrm{H})$ Later cleavage stage tudor $^{\mathrm{WC}} / \mathrm{Df}(2 \mathrm{R}) \mathrm{PL} 3$ embryo. Label is becoming more diffuse. (I) tudor $^{\mathrm{WC}} / \mathrm{Df}(2 \mathrm{R}) \mathrm{PL} 3$ embryo in the process of forming pole buds. Some localized label is present. (J) Slightly later stage tudor ${ }^{\mathrm{WCO}} / \mathrm{Df}(2 \mathrm{R}) \mathrm{PL} 3$ embryo than in (I). The blastoderm is about to form. Pole buds are not present. A very faint localized label is present at the posterior embryo pole. (K) tudor $^{\mathrm{WC}} / \mathrm{Df}(2 \mathrm{R}) \mathrm{PL} 3$ embryo beginning gastrulation. Pole cells are not present. Localized posterior label and the general background staining are absent.

forming a complex with nanos which becomes localized to the anterior end in a Bicaudal-D embryo. If this is correct, vasa should be localized to the anterior pole as well as to the posterior pole in Bicaudal-D embryos. This hypothesis has not been borne out; under egglaying conditions in which Bicaudal-D females produce large numbers of embryos with a bicaudal phenotype, we see no localization of the vasa protein to the anterior pole of oocytes, in vitro activated eggs or early cleavage stage embryos. A failure to see vasa localization to the anterior pole of Bicaudal-D embryos has been independently noted by Wharton and Struhl (1989). Since vasa distribution in a Bicaudal-D embryo is normal, it is not the case that the formation of the anterior abdomen is initiated via a mislocalization of vasa, and the bicaudal egg must increase the concentration of posterior rescuing activity (nanos product) at the anterior end independent of vasa localization. Given that formation of the anterior end still requires vasa activity, vasa must have a function other than simply localizing nanos (see discussion).

\section{Discussion}

Polar granules are concentrated at the posterior pole of late stage oocytes and early embryos, and may be involved in the posterior localization and function of germ cell determinants and activities that specify abdominal segmentation. We have shown previously that the vasa protein is an essential component of the polar granule (Hay et al. 1988a,b). In an attempt to study the mechanism and function of polar granule localization, 
we have followed the fate of the vasa product in six maternal effect mutants, vasa, staufen, oskar, valois, tudor, and Bicaudal-D, because these mutations alter localization of cytoplasmic determinants required for development of the germ line and for embryonic abdomen formation.

\section{Functions of vasa}

The phenotype of vasa is complex. A hypomorphic allele, vasa ${ }^{\mathrm{PD}}$, has vasa immunoreactivity restricted largely to the germarium, and fails to localize (or perhaps even assemble) polar granules. In contrast, null mutants are usually blocked during early oogenesis at roughly the stages in which vasa seems to be produced in vasa ${ }^{\mathrm{PD}}$. These results suggest a requirement for vasa at two distinct phases of oogenesis. Early during oogenesis vasa is necessary for the development of the oocyte and nurse cells following their formation, whereas in the second half of oogenesis vasa is required for the localization of cytoplasmic determinants required for germ line formation and abdomen specification. During embryogenesis, there may be additional requirements for vasa. At the stage of the somatic blastoderm formation, vasa ${ }^{\mathrm{PD}}$ and vasa ${ }^{\mathrm{DL}}$ embryos show frequent cellularization defects (data not shown). This may reflect an essential function of the low level of vasa seen throughout the wildtype embryo, as revealed in immunocytochemical experiments.

The vasa protein has a high degree of homology with members of the helicase class of RNA-binding proteins (Hay et al. 1988; Lasko and Ashburner, 1988), suggesting that it functions in some context as an RNAbinding protein. Three possible roles that vasa as an RNA-binding protein might be playing in the function of the posterior determinant system are (1) to regulate the translation of localized transcripts required for determination of posterior structures; (2) to participate in the localization of the posterior determinants by providing a permissive environment for this localization (its helicase activity stabilizing a particular transcript conformation that allows binding by specific RNAbinding proteins) or (3) to localize the determinants directly. The last possibility is unlikely in light of the vasa localization phenotype in Bicaudal- $D$ embryos: females transheterozygous for vasa and Bicaudal-D suppress the bicaudal phenotype (anterior function of abdominal determinants) though vasa is not localized anteriorly in Bicaudal-D embryos. We cannot completely rule out the possibility that vasa is required for directly targeting determinants to the posterior pole in the normal embryo, but that it plays a different role in localization via a Bicaudal-D-dependent mechanism. The first two possibilities can be distinguished on the basis of the localization of the nanos product in Bicaudal- $D$ vasa transheterozygote embryos. Since embryos from heterozygous vasa females, which contain large amounts of vasa localized to the posterior pole, give rise to normal posterior structures while the anterior formation of abdominal structures is suppressed in transheterozygotes of vasa and Bicaudal-D, it is possible that the vasa protein present throughout the somatic region of the cleavage stage embryo provides the vasa-dependent activity present in Bicaudal-D embryos. Decreasing the level of vasa in transheterozygotes reduces the amount of available anterior vasa below a critical threshold and thus prevents anterior abdomen formation.

\section{Localization of vasa}

Following fertilization or egg activation polar granules undergo rapid changes in their associations with other structures and in their morphology. During oogenesis polar granules are associated with mitochondria and appear to contain large amounts of RNA. Following either egg activation or fertilization, they lose this association with mitochondria, fragment and become associated with polysomes (Mahowald, 1962, 1968, $1971 b$ ). These observations raise the possibility that different mediators of localization, including perhaps cytoskeletal elements, may be used during oogenesis and early embryogenesis.

The process of vasa localization is altered at an early stage by oskar and staufen mutations. Females homozygous for strong alleles of staufen and oskar synthesize roughly normal amounts of vasa though it does not become localized during oogenesis. Thus both oskar and staufen gene products are required for some aspect of the initial vasa localization event. Further evidence for this hypothesis derives from studies of $o s k a r^{301}$, the temperature-sensitive allele. The temperature-sensitive period for the oskar-dependent development of the abdominal deletion phenotype corresponds to the last half of oogenesis (stages 10-14), the same time period during which polar granules become localized to the posterior oocyte pole (Lehmann and Nüsslein-Volhard, 1986). Indeed, some vasa is localized at permissive, but not at nonpermissive, temperatures during oogenesis. Thus the strength of the oskar phenotype is related to the amount of vasa, and probably polar granules, that become localized to the posterior pole. The oskar activity in oskar ${ }^{301}$ mutants is not normal even at the permissive temperature since pole cells do not form in these embryos and the vasa that is localized to the posterior pole during oogenesis does not remain localized following oviposition. The latter observation suggests that oskar-dependent activity (which may be either quantitatively or qualitatively insufficient in the mutant) is also required during cleavage stages of embryogenesis to maintain vasa localization.

Though mutations of oskar, staufen, cappuccino and spire have a similar vasa localization phenotype (this paper; Manseau and Schüpbach, 1989) they differ in their interactions with Bicaudal-D. Bicaudal-D in a homozygous oskar background suppresses the bicaudal phenotype (Lehmann and Nüsslein-Volhard, 1986), while Bicaudal-D in a heterozygous staufen background (Mohler and Wieschaus, 1986) or a homozygous cappuccino or spire background (Manseau and Schüpbach, 1989) results in an enhancement of the bicaudal phenotype.

If the product of the oskar locus is a polar granule component having a similar distribution to that of vasa 
and this product is required for some aspect of abdominal determinant (nanos) function, the block in anterior function of the abdominal determinants could be similarly explained. Oskar product function may be required for nanos function either indirectly, through interactions with vasa, or directly through association with the products of the nanos or Bicaudal-D loci. In the case of staufen, cappuccino and spire, a likely possibility is that these gene functions are required for transport of the abdomen-specifying signal (nanos) to the posterior pole. In the absence of any of these gene functions, posterior localization of the abdomen-inducing signal does not occur. In Bicaudal-D embryos, some of this activity becomes localized to the anterior pole under the influence of the Bicaudal-D-dependent localization mechanism. In the double mutant combinations of Bicaudal-D with staufen, cappuccino or spire described above, more of this activity becomes localized to the anterior pole because the action of the Bicaudal-D-dependent localization mechanism is not competing as much or at all (for nanos-binding perhaps) with elements required for normal posterior-dependent localization.

The phenotype of tudor is more difficult to interpret, since tudor ${ }^{\mathrm{wC}}$, a strong allele of tudor, is almost certainly hypomorphic. Boswell and Mahowald (1985) have shown in a series of tudor mutants that the number and size of structures tentatively identified as polar granules, based on their electron-dense appearance and association with mitochondria, decreases as the severity of the abdominal deletion phenotype increases. Polar granules are not apparent in tudor ${ }^{\mathrm{WC}} / \mathrm{Df}$ (2L)PL3 heterozygotes (Boswell and Mahowald, 1985), yet we see good vasa localization during oogenesis and during early cleavage stages following fertilization. On the other hand, consistent with this phenotypic series, we find that in $t u d o r^{2}$, a weaker tudor allele, the localized vasa immunoreactivity typically is more abundant than in tudor $^{\mathrm{WC}} / \mathrm{Df}(2 \mathrm{~L}) \mathrm{PL} 3$ heterozygotes. It is not clear from this phenotypic series, however, whether vasa would be localized in oocytes from mothers totally deficient in tudor function. One possibility is that the strong phenotype (if viable) is a complete lack of localization. Alternatively, or perhaps in addition to this aspect of tudor function, tudor may play some other role in polar granule function following fertilization. This seems likely because, though vasa localization is quite good in early cleavage stage tudor embryos, and vasa immunoreactivity remains somewhat localized until about the time the pole cells would normally be forming, pole cells do not form and abdominal segmentation is disrupted to a variable extent. The fact that vasa is well localized in oocytes and early embryos from tudor $^{\text {WC8 }} / \mathrm{Df}(2 \mathrm{~L})$ PL 3 females, but that localized polar granules are not visible with electron microscopy suggests that the tudor product is a polar granule component required for interactions between granule components necessary for assembly of a functional granule structure. The observation that transheterozygotes of tudor and Bicaudal-D suppress the bicaudal phenotype, as is the case with vasa, is also consistent with the idea that tudor encodes a polar granule component required for the function of the posterior determinants. The observation that the abdominal segment deletion phenotype is variable in tudor ${ }^{\mathrm{wC}}$, whereas pole cells never form, may simply reflect a higher threshold requirement for tudor-dependent functions for pole cell formation, or it may indicate a qualitative difference in the mutant's ability to carry out functions required for abdomen and pole cell development.

valois $^{\mathrm{PE}}$ appears to be a strong allele of valois, based on the consistency of both the abdominal deletion and vasa localization phenotypes, either as a homozygote or in heterozygous combination with a deficiency for the region. It shows good localization during oogenesis, but this disappears very rapidly following fertilization, even though normal amounts of vasa protein can be detected on Western blots. This sugests that the maternal valois product is required for some aspect of stabilization of vasa localization following fertilization. Interestingly, many valois embryos do not cellularize, or cellularize incompletely. This phenotype is consistent with the possibility that valois might be important for some aspects of cellular function (the cytoskeleton perhaps) in the embryo which are required for both somatic cellularization and maintenance of polar granule localization. We cannot completely rule out a role for valois during the initial localization process.

\section{Summary}

In summary, it seems likely that, based on vasa's homology with RNA helicases, its localization phenotype in Bicaudal-D and its suppression of the bicaudal phenotype, a primary role of vasa in the function of the posterior determinant system is to regulate the translation of mRNA important for determination of posterior structures and/or to provide a permissive environment (RNA secondary structure) for RNA localization via other RNA-binding proteins. The fact that localized polar granules are absent in vasa mutants indicates that vasa is also a necessary component of the granules. Vasa's role during early oogenesis is mysterious.

The vasa localization phenotypes described for the other six posterior group genes show that function of these genes is required for posterior vasa localization during oogenesis (oskar, staufen, cappuccino and spire), its maintenance following fertilization (valois), or events occurring after fertilization required for macroscopic polar granule structure and posterior determinant functions (tudor). Mutations in staufen, cappuccino, spire and oskar all affect early steps of vasa localization in oocytes. Their different interactions with Bicaudal-D, however, suggest that staufen, cappuccino and spire gene functions are likely to be required for transport of determinants to the posterior pole or their localization there, while oskar activity, perhaps in the form of another polar granule component or a molecule required for posterior-specific localization of the gran- 
ules, is required for proper function of the determinants. In addition, oskar-dependent activity may also be required for maintenance of posterior vasa localization following fertilization. The observation that valois mutations affect somatic cellularization and the maintenance of vasa localization following fertilization suggest that the valois product functions as a cytoskeletal component required throughout the embryo following fertilization. In contrast, tudor function may not be required for vasa localization during oogenesis or the maintenance of this localization following fertilization since vasa remains localized through late cleavage stages of embryogenesis in tudor embryos. This observation and the lack of macroscopic polar granules seen in tudor embryos suggest that the tudor product is a polar granule component that is required for the assembly of that structure. Since tudor embryos have abdominal segment deletions and lack pole cells though vasa is localized relatively normally, and tudor suppresses Bicaudal-D, tudor may be required for the activation of the posterior determinant activity or to provide an environment permissive for the localization of this activity.

We would like to thank Trudi Schüpbach for providing stocks of vasa (vasa ${ }^{\mathrm{PD} 23}$ and vasa ${ }^{\mathrm{DL}}$ ), tudor (tudor ${ }^{\mathrm{WCB}}$ ), a deficiency that uncovers the tudor locus (Df(2L) PL3), staufen (staufen ${ }^{\mathrm{HLS}}$ ), a deficiency that uncovers the staufen locus (Df(2L) PC4), valois (valois ${ }^{\mathrm{RB}}$ ), and a deficiency that uncovers the valois locus (Df(2L)TW2), Robert Boswell for providing stocks of tudor (tudor ${ }^{2}$, tudor $^{3}$, tudor $^{4}$ ), and Ruth Lehmann for stocks of the two deficiencies Df(2L)A267 and Df(2L)TE116-GW18, which in heterozygous combination delete part of the vasa locus, staufen (staufen ${ }^{\mathrm{D} 3}$ ), oskar $\left(o s k a r^{166}, o s k a r^{336}, o s k a r^{301}\right)$, a deficiency that uncovers the oskar locus, stocks of valois (valois ${ }^{\mathrm{PE}}$ ), and stocks of $B i$ caudal-D (Bicaudal-D $D^{7134}$, Bicaudal-D $D^{\mathrm{IIE}}$ ). We also thank Robert Caretto for help with some of the antibody stainings, and Larry Ackerman and Tina Chueng for their help with the photography and artwork. B. $\mathrm{H}$. is supported by an NIH training grant and L. Y. J. and Y. N. J. are Howard Hughes Investigators.

\section{References}

Boswell, R. E. And Mahowald, A. P. (1985). tudor, a gene required for assembly of the germ plasm in Drosophila melanogaster. Cell 43, 97-104.

Davidson, E. (1986). Gene Activity in Early Development. New York: Academic Press.

Dieckmann, C. L. and Tzagolofp, A. (1985). Assembly of the mitochondrial membrane system: CBP6, a yeast nuclear gene necessary for the synthesis of cytochrome $b^{*}$. J. biol. Chem. 260 , $1513-1520$.

Driever, W. and NÚsslein-Volhard, C. (1988). A gradient of bicoid protein in Drosophila embryos. Cell 54, 83-93.

Frohnhofer, H. G., Lehmann, R. and Nússlein-Volhard, C. (1986). Manipulating the anterior-posterior pattern of the Drosophila embryo. J. Embryol. exp. Morph. 97 Supplement, 169-179.

Gutzert, H. O. AND Koppa, R. (1982). Time-lapse film analysis of cytoplasmic streaming during late oogenesis of Drosophila. J. Embryol. exp. Morph. 67, 101-111.

Hay, B., Ackerman, L., Barbel, S., Jan, L. and Jan, Y. N. (1988a). Identification of a component of Drosophila polar granules. Development 103, 625-640.
HAY, B., JAN, L. Y. AND JAN, Y. N. (1988b). A protein component of Drosophila polar granules is endoded by vasa and has extensive sequence similarity to ATP-dependent helicases. Cell 55, 577-587.

Hülskamp, M., Schroder, C., Pfeifle, C., Jäckle, H. and Tautz D. (1989). Posterior segmentation of the Drosophila embryo in the absence of a maternal posterior organizer gene. Nature 338, $629-632$.

Illmensee, K., Mahowald, A. P. and Loomis, M. R. (1976). The ontogeny of the germ plasm during oogenesis in Drosophila. Devl Biol. 49, 40-65.

Irish, V., Lehmann, R. And Akam, M. (1989). The Drosophila posterior-group gene nanos functions by repressing hunchback activity. Nature 338, 646-648.

King, R. C. (1970). Ovarian Development in Drosophila melanogaster. New York: Academic Press.

Kunkel, T. A., Roberts, J. D. And Zakour, R. A. (1987). Rapid and efficient site-specific mutagenesis without phenotypic selection. Methods in Enzymology 154, 367-382.

LAEMMLI, U. K. (1970). Cleavage of structural proteins during the assembly of the head of bacteriophage T4. Nature $227,680-685$.

Lasko, P. F. and Ashburner, M. (1988). The product of the Drosophila gene vasa is very similar to eukaryotic initiation factor-4A. Nature 335, 611-617.

Lehmann, R. and Nusslein-Volhard, C. (1986). Abdominal segmentation, pole cell formation, and embryonic polarity require the localized activity of oskar, a maternal gene in Drosophila. Cell 47, 141-152.

Mahowald, A. P. (1962). Fine structure of pole cells and polar granules in Drosophila melanogaster. J. exp. Zool. 151, 201-216.

Mahowald, A. P. (1968). Polar granules of Drosophila. II. Ultrastructural changes during early embryogenesis. J. exp. Zool 167, 237-262.

Mahowald, A. P. (1971a). Polar granules of Drosophila. III. The continuity of polar granules during the life cycle of Drosophila. J. exp. Zool. 176, 329-344.

Mahowald, A. P. (1971b). Polar granules of Drosophila. IV. Cytochemical studies showing loss of RNA from polar granules during early embryogenesis. J. exp. Zool. 176, 345-352.

Mahowald, A. P., Goralski, T. J. and Caulton, J. H. (1983). In vitro activation of Drosophila eggs. Devl Biol. 98, 437-445.

Maniatis, T., Fritsch, E. F. and Sambrook, J. (1982). Molecular Cloning: A Laboratory Manual. Cold Spring Harbour, New York: Cold Spring Harbour Laboratory.

MANSEAU, L. AND SCHUPBACH, T. (1989). cappuccino and spire: two unique maternal-effect loci required for both the anteroposterior and dorsoventral patterns of the Drosophila embryo. Genes and Dev. 3, 1437-1452.

Mohler, J. and Wieschaus, E. F. (1986). Dominant maternaleffect mutations of Drosophila melanogaster causing the production of double abdomen embryos. Genetics $112,803-822$.

Nikı, Y. (1986). Germline autonomous sterility of the P-M dysgenesis hybrids and their application to germline transfers in Drosophla melanogaster. Devl Biol. 113, 255-258.

Rio, D. C., LASki, F. A. And Rubin, G. M. (1986). Identification and immunochemical analysis of biologically active Drosophila $\mathrm{P}$ element transposase. Cell 44, 21-32.

Sander, K. ANd Lehmann, R. (1988). Drosophila nurse cells produce a posterior signal required for embryonic segmentation and polarity. Nature 335, 68-70.

SchúpbaCh, T. AND WIESChaUS, E. (1986). Maternal-effect mutations altering the anterior-posterior pattern of the Drosophila embryo. Wilhelm Roux's Arch. dev. Biol. 195, 302-317.

STRUHL, G. (1989). Differing strategies for organizing anterior and posterior body pattern in Drosophila embryos. Nature 338 , $741-744$.

Wharton, R. P. and Struhl, G. (1989). Structure of the Drosophila BicaudalD protein and its role in localizing the posterior determinant nanos. Cell 59, 881-892. 